\title{
Anforderungstaxonomie für industrielle Cloud Infrastrukturen durch Internet of Things- und Big Data- Applikationen
}

\author{
Kornelia Schuba, Carsten Pieper, Sebastian Schriegel, Khaled Al-Gumaei \\ Fraunhofer IOSB-INA \\ Langenbruch 6 \\ 32657 Lemgo \\ kornelia.schubaliosb-ina. fraunhofer.de \\ carsten.pieper@iosb-ina. fraunhofer.de \\ sebastian.schriegeldiosb-ina.fraunhofer.de \\ khaled.al-gumaeidiosb-ina.fraunhofer.de
}

\begin{abstract}
Zusammenfassung. Die Anzahl miteinander vernetzter Sensoren, Geräte und Systeme wird in den nächsten Jahren weltweit weiter massiv ansteigen. Die Erhebung dieser Menge an Daten, es wird von Big Data gesprochen, ist nur dann sinnvoll, wenn Schlussfolgerungen daraus extrahiert werden. Auch die Anwendungsbereiche industrielle Automation und Smart City werden von Internet of Things- (IoT) und Big Data-Technologie derzeit maßgebend geprägt. Mit dem Anstieg von Datenquellen (IoT) und somit erzeugten Daten, die verarbeitet und analysiert werden müssen, müssen IT-Infrastrukturen entwickelt und angewandt werden, die die Anforderungen im Zusammenhang mit Big Data und IoT erfüllen. Eine Möglichkeit die wachsenden Datenmengen und Anforderungen zu bewältigen bietet die Integration von IoT und Big Data in Cloud Infrastrukturen. Heute sind viele Cloud-Lösungen marktverfügbar und die Begriffe IoT und Big Data werden inflationär genutzt, daher fällt es zunehmend schwer eine passende Lösung auszuwählen, die spezifischen Anforderungen einer Anwendung genügen. In diesem Paper wird eine strukturierte Anforderungstaxonomie entwickelt, die die spezifischen industriellen Anforderungen hervorhebt und die es erleichtert Cloud Plattformen passend zur Applikation auszuwählen und zu konfigurieren. Die Taxonomie wird an einem Use Case aus der industriellen Produktion und einem Use Case aus dem Smart City-Bereich evaluiert.
\end{abstract}

\section{Industrial Internet of Things und die Notwendigkeit einer Anforderungstaxonomie}

Die Anzahl an vernetzten Geräten ist in den letzten Jahren stark gestiegen und dieser Trend wird nicht abreißen. Ebenso wird die Nutzung von Internet of Things in der industriellen Automation, dem Industrial Internet of Things (IIoT) erheblich voranschreiten und zunehmend an Bedeutung gewinnen [1]. Dementsprechend müssen auch die mit dem IoT einhergehenden Technologien, wie Big Data und Cloud Computing entsprechend der Anforderungen der Industrie 4.0 evaluiert und angepasst werden [1]. Die 
Einbindung von Sensoren und Aktoren hat zur Folge, dass große Datenmengen (Big Data) erzeugt, aufgezeichnet und analysiert werden müssen. Als Folge dessen ist die Integration von Cloudstrukturen in die Produktionsumgebung zu sehen. Dieser strukturelle Wandel von der Automatisierungspyramide, die im Umfeld Industrie 3.0 angesiedelt ist, zur IIoT-Architektur im Umfeld der Industrie 4.0 ist in Abbildung 1 dargestellt.
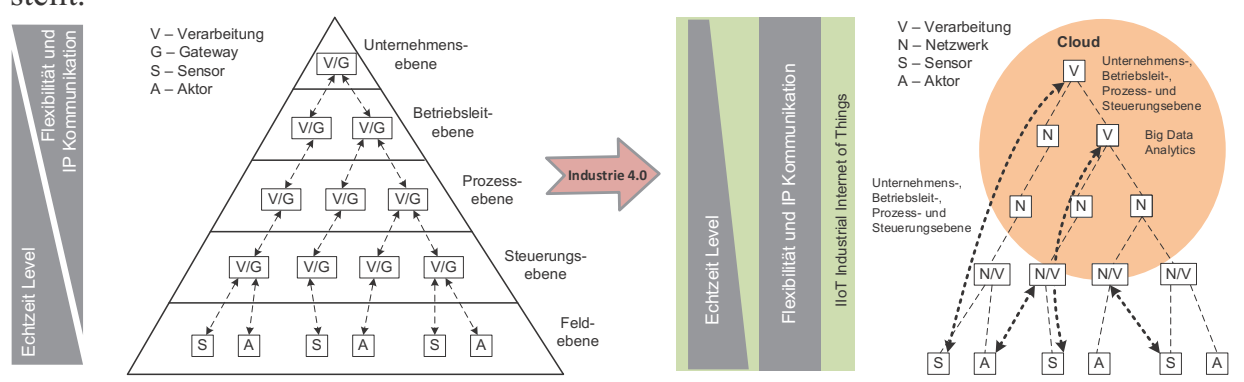

Abb. 1. Von der Automatisierungspyramide zur Industrie 4.0 Architektur IIoT (vgl. [2])

Die Architektur der Automatisierungspyramide ist in fünf Ebenen, die Feld-, Steuerungs-, Prozess-, Betriebsleit-, und Unternehmensebene gegliedert. Die einzelnen Ebenen sind durch Gateways verbunden, die manuell konfiguriert werden müssen, was lediglich im Rahmen einer (undynamischen) Massenproduktion effizient ist. Eine flexible und IP -basierte Kommunikation ist hauptsächlich in den höheren Ebenen, eine Echtzeitübertragung eher in den unteren Ebenen der Pyramide zu finden. Die Industrie 4.0 definiert zu effizienter Massenproduktion zusätzliche Anforderungen an die Produktionstechnik: flexibilisierte Massenproduktionen, automatisierte Produktionen bis zur Losgröße eins und eine durch Daten getriebene (Big Data) Effizienzoptimierung (Condition Monitoring, Predictive Maintenance, Optimierung) [1, 3]. Die Industrie 4.0 Architektur IIoT, die als Basis dafür dienen soll, ist losgelöst von Ebenen und starrer Kommunikation zwischen diesen. IIoT setzt dazu unter Anderem viel auf die Nutzung von Virtualisierung und Cloud-Technologie. Ausgenommen der Feldebene, können alle für die Automation notwendigen Funktionen sowohl innerhalb als auch außerhalb der Cloud implementiert werden. Die bereits angesprochenen Datenmengen (Big Data) und deren Analyse kann z. B. effizient als Cloud-Service umgesetzt werden. Insgesamt sind deutlich weniger Gateways im Einsatz was eine IP-basierte flexible Kommunikation vom Sensor bis in die Cloud durchgängig möglich macht. Die Echtzeit-Fähigkeit in den oberen Ebenen (IT und Smart Services) soll mit dieser Architektur ebenfalls gesteigert werden [2].

Insbesondere die Integration von Cloud Technologien ist Thema in diesem Paper. Von dem Cloud Service müssen Anforderungen, die die Industrie stellt, erfüllt werden um z.B. Produktionsabläufe, Reaktionszeiten, Sicherheitsaspekte und Verfügbarkeit einhalten zu können. Diese Anforderungen werden in einer Anforderungstaxonomie zusammengestellt und evaluiert. Im Stand der Technik werden die zentralen Begriffe IoT/ IIoT, Big Data und Cloud Computing erläutert und in Zusammenhang gestellt. Anschließend werden zunächst die industriellen Anforderungen an eine Cloud beschrieben und schließlich diese Anforderungen in Bezug auf ihre Bedeutung für die 
Industrie in der Anforderungstaxonomie bewertet und evaluiert. Dann wird die Anforderungstaxonomie anhand von zwei Bespielen auf ihre Plausibilität geprüft. Den Abschluss bildet eine Zusammenfassung der Erkenntnisse und Resultate der Untersuchung und Taxonomie, die auch einen Ausblick auf weitere mögliche Fragestellungen und Forschungsansätze gewährt.

\section{Stand der Technik: IoT, Big Data und Cloud Computing}

In der vernetzten Welt sind die drei Begriffe IoT, Big Data und Cloud unabdingbar und werden häufig zusammen verwendet. Dies ist der Kausalität der drei Themen geschuldet. Viele vernetzte IoT Geräte erzeugen viele und unstrukturierte Daten (Big Data), die wiederum in Cloud Plattformen gespeichert und ausgewertet werden können, in denen Speicherkapazität und Rechenleistung beliebig gebucht werden können.

IoT: Der Begriff Internet of Things (IoT) wird als Infrastruktur von vernetzten Sensoren, Geräten oder Systemen definiert, die sowohl auf Informationen aus der physikalischen und der virtuellen Welt reagieren. Die Verknüpfung dieser beiden Welten ermöglicht es neue intelligente Lösungen in der physikalischen Welt zu schaffen. Die Anwendungsbereiche von IoT erstrecken sich von Smart Home über Smart City, den Agrar- und Gesundheitsbereich bis hin zur industriellen Automation [4]. In der industriellen Automation wird der Begriff Industrial Internet of Things (IIoT) verwendet; hier werden smarte Objekte und Maschinen vernetzt und somit die Produktion „smart“ gestaltet $[5,6]$. Der ausgeprägte Anstieg an vernetzten Geräten und auch Maschinen wird die Menge an Daten, die verarbeitet werden müssen weiterhin deutlich erhöhen, daher ist IoT bzw. IIoT eine der Hauptquellen von Big Data [7].

Big Data: Big Data wird oft durch die Charakteristika volume (große Datenmengen), variety (verschiedenste Datenquellen), velocity (enormer und kontinuierlicher Datenfluss) und variability (Veränderung der Daten, wie Format oder Qualität) beschrieben, die als Basis für eine skalierbare Architektur dienen [8, 9]. Im Themenfeld Big Data spielt die Datenanalyse eine wichtige Rolle, denn die Daten müssen insbesondere für industrielle Anwendungen mit Echtzeitbezug direkt analysiert und in Bezug auf bisherige Daten gedeutet werden. Mit dem Anstieg von Datenquellen und somit erzeugten Daten, die verarbeitet und analysiert werden müssen, müssen ITInfrastrukturen gewählt werden, die die Anforderungen im Zusammenhang mit Big Data und IoT erfüllen. Die Integration von IoT und Big Data in Cloud Infrastrukturen, die die Möglichkeit der virtuell unbegrenzten Kapazitäten von z. B. Speicher, Prozessoren und Leistung haben, bietet eine Lösung, die wachsenden Datenmengen und damit verbundenen Anforderungen zu bewältigen [10]. Cloud Computing Plattformen lösen diese Problematik oft durch angebotene Analysetools und Datenbanken, die auf die Speicherung und das Handling mit Big Data ausgelegt sind.

Cloud Computing: Cloud Computing bezeichnet ein Modell, das on-demand Netzwerkzugriffe auf im Kollektiv genutzte Datenressourcen liefert. Datenressourcen können in dem Fall Netzwerke, Speicher, Server oder Anwendungen sein, die schnell bereitgestellt werden und einen geringen Verwaltungsaufwand haben können [8]. Die 
Cloud Computing Architektur kann in drei Service Modelle unterschieden werden: Infrastructure as a Service (IaaS), Platform as a Service (PaaS), Software as a Service (SaaS). Dabei werden im IaaS-Modell dem Anwender Prozessoren, Speicher, Netzwerke und Computer-Ressourcen bereitgestellt. Der Anwender kann diese nutzen und beliebige Software, inklusive Applikationen und Betriebssystem, einsetzten und installieren. Insgesamt hat der Anwender Kontrolle über Betriebssystem, Speicher, entwickelte Applikationen und eingeschränkt auch auf Netzwerkkomponenten, wie z.B. Firewall. Keinen Zugriff auf die Infrastruktur, wie Speicher, Netzwerk und Server hat der Anwender dahingegen beim PaaS-Modell. In diesem Fall werden vom Anwender lediglich mit angebotenen Programmiersprachen, Services und Tools erstellte oder erworbene Applikationen genutzt. Bei Nutzung des SaaS-Modells kann der Anwender nur Anwendungen und Applikationen auf der Cloudinfrastruktur des Anbieters nutzen. Die Infrastruktur einer Cloud kann als private, public, community oder hybrid Cloud strukturiert sein. In der privaten Cloud wird die Infrastruktur exklusiv für eine Organisation bereitgestellt, der die Cloud Infrastruktur gehören und auch von dieser gemanagt und gesteuert werden kann. Diese Aufgabe kann auch an Dritte weitergegeben werden oder in einer Kombination aus beidem zusammenlaufen. Insgesamt kann die private Cloud On- oder Off-Premise existieren. Das bedeutet, dass die Hardware, wie Server, Netzwerke etc. lokal (z.B. in einem eigenen Rechenzentrum) bereitstehen. Off-Premise dahingegen bezeichnet die Auslagerung der Hardware in dem Sinne, dass z. B. die Infrastruktur von einem Anbieter gemietet wird. Die public Cloud Infrastruktur kann von der Allgemeinheit offen genutzt werden. Im Gegensatz zur private Cloud kann die public Cloud nicht vom User selbst gemanagt und gesteuert werden, dies wird von einer Business-, Akademischen- oder Regierungsorganisation übernommen. Die public Cloud existiert auf der Basis der Infrastruktur des Cloud Anbieters. Des Weiteren kann die Cloud für eine bestimmte Gruppe/ Community bereitgestellt werden, die Interessen oder Themengebiete gemeinsam teilen. Die community Cloud kann, wie die private Cloud, sowohl on- als auch off-Premise bestehen. Letztlich kann auch eine Kombination von mindestens zwei der drei vorgestellten Deployment Modelle, das hybrid Cloud Modell umgesetzt werden. Dieses bietet die Möglichkeit z. B. je nach Anwendung und deren Anforderung zwischen der private und public Cloud zu entscheiden [8].

\section{$3 \quad$ Herleitung einer Anforderungstaxonomie}

In der Anforderungstaxonomie an industrielle Cloud Infrastrukturen werden hier zum einen die technischen Detailanforderungen an die Cloud und zum anderen die Anwendungsfälle der Industrie selbst betrachtet. Diese Anwendungen können in die Basisapplikationstypen Monitoring (Beobachten) und Control (Steuern) struktuiert werden. Der Bereich Monitoring enthält die Betrachtung von z.B. gerichtsverwertbaren und historischen, als auch von live Daten, Predictive Maintenance, Condition Monitoring und Security. Das Themenfeld Control beinhaltet die Steuerung von Prozessen. Diese Steuerung kann zeitunkritisch oder zeitkritisch und/ oder sicherheitsrelevant sein. Der Sicherheitsaspekt bezieht sich sowohl auf Safety als auch auf Security. Die Kommunikation zwischen Maschinen und Sensoren und auch Optimierungsprozesse in der 
industriellen Produktion sind dem Bereich Control zugeordnet. Bringt man den Aspekt Monitoring und Control zusammen, ergibt sich eine Anforderung der Industrie, die sich durch die Kombination beider Bereiche als besonders hohe Anforderung darstellt. Es handelt sich um ein Monitoring auf Grund dessen eine Steuerung der Maschine oder eine Handlungsanweisung an einen Menschen gestellt wird. Beiden Bereichen wird ebenfalls der Anwendungsfall Security zugeordnet, da dieses sowohl in Zusammenhang mit Datenaufnahme und Überwachung als auch im Bereich der Steuerung relevant ist. Insgesamt zeigen diese Basisanwendungsfälle der Industrie deren Breite und Heterogenität.

Vorbereitend auf die Anforderungstaxonomie wurden die technischen und generischen Detailanforderungen an die Cloudlösung definiert. Zwei davon sind die Einbindung von IoT als auch Big Data in die Cloud Struktur. Diese beiden Anforderungen haben Verzahnungen mit weitere Anforderungen, die im Folgenden beschrieben werden und dabei eine Grundvoraussetzung an das Cloud System darstellen. Bei vielen Anforderungen sind Korrelationen untereinander festzustellen.

Skalierbarkeit und Flexibilität: Es muss die Möglichkeit bestehen jederzeit Ressourcen zu erweitern, je nachdem wie sie verwendet und gebraucht werden. Dies kann sich beispielsweise auf Rechenleistung, Anzahl an IoT-Geräten oder auch Speichervolumen beziehen. Eine Flexibilität der Cloud muss im Bereich der flexiblen und vernetzten Produktion gegeben sein. Dies kann z.B. bedeuten, dass ein Prozess, der on-premise gelaufen ist, nun off-premise verschoben werden soll; dies muss dann mit der Cloud umgesetzt werden können.

Quality of Service (QoS): In der industriellen Automation fällt unter QoS insbesondere eine kurze Latenzzeit als Anforderung an die Systeme. Zum Beispiel wird in den Bereichen Motion Control 250 $\mu \mathrm{s}-1 \mathrm{~ms}$, Condition Monitoring 100ms und Augmented Reality 10ms Latenzzeit gefordert [11]. Eine Echtzeit-Verarbeitung, die von der Latenzzeit abhängt, wird z. B. für Stream Processing benötigt. Diese Echtzeit-Kontrolle und auch Steuerung muss von einer industriellen Cloud zur Verfügung gestellt werden.

Preismodell: Ein Preismodell einer Cloud muss, wie die Cloud selbst, flexibel und skalierbar sein. Und vor allem sollten nur die Leistungen abgerechnet werden, die auch gebraucht/ in Anspruch genommen werden. Denn eine flexible Produktion ist nur bis zu einem gewissen Grad berechenbar und dementsprechend auch die benötigte Dimension der Infrastruktur. Viele Cloud Anbieter bieten ihre Plattformen nach dem Preismodell pay-as-you-go an, dies bedeutet, nur für das zu bezahlen, das auch benutzt wurde. Andere Preismodelle schnüren Pakete mit angebotenen Leistungen und z.B. vorgegebener Datenmenge, die beliebig zusammengestellt werden können oder haben Grundpakete, die die Basis des Systems beschreiben und lediglich zwischen einer beschränkten Anzahl an verschiedenen Ausführungen wählbar sind.

Sicherheit: Diese Anforderung muss in der industriellen Produktion ein wichtiger Punkt. Die Absicht produktionstechnische und durchaus sensible Daten in der Cloud zu speichern erfordert, ein Sicherheitskonzept um vor Cyber Angriffen zu schützen.

Service Level Agreement (SLA): Kriterien wie die Verlässlichkeit, Verfügbarkeit und Leistungsspektrum werden über das SLA definiert und garantiert. Auf diese Weise 
kann die Wahrscheinlichkeit des Risikos ermittelt werden, mit der z. B. die Produktion zum Erliegen kommen könnte bzw. in welchem Zeitraum Server- oder ähnliche Probleme gelöst werden. Über das SLA werden auch Ausfallentschädigungen deklariert.

Analyse: In der industriellen Cloud muss die Analyse von unterschiedlichsten Datentypen und -mengen (unter anderem Big Data) gewährleistet werden. Diese Analyse muss in bestimmten Anwendungsbereichen in Echtzeit umgesetzt werden. Cloud Anbieter integrieren verschiede Analysetools, die z.B. Anomalie Erkennung oder Maschine Learning Komponenten enthalten.

Kommunikation, Protokolle, Plug and Play: Im Rahmen der Vernetzung im industriellen Umfeld und der Interaktion zwischen Maschine zu Maschine ist es notwendig, dass Protokolle miteinander interoperabel sind. Entsprechend wird die Anforderung einer hohen Bandbreite an unterstützten Protokollen an die Cloud Plattform gestellt [12]. Das übergeordnete Ziel ist es, dass eine Integration in bestehende Systeme simpel und schnell zu realisieren ist, ganz nach dem Prinzip Plug and Play.

Blockchain: Die Blockchain-Technologie bietet eine Möglichkeit Informationen unverfälschbar und so vertragssicher zu speichern.

Safety: Daten/ Information müssen verfälschungssicher (SIL-Level) übertragen und gespeichert werden um Safety-relevante Prozesse damit zu steuern.

\subsection{Taxonomie}

Anhand der aufgestellten Anforderungen wurde eine Taxonomie erstellt, die in Abbildung 2 dargestellt ist. Hierbei sind die Basisanwendungsfälle der Industrie, wie für eine Taxonomie üblich in einer Baumstruktur abgebildet. Dabei wurden die Anwendungsfälle entsprechend ihres Bedarf an Echtzeitverarbeitung strukturiert: der Bedarf fällt von dem überlappenden Bereich von Control und Monitoring nach außen hin. Eine Ausnahme ist der Anwendungsfall Security, der sowohl für Control als auch Monitoring gilt und nicht diesem Ordnungsprinzip folgend angeordnet wurde. Die technischen und generischen Detailanforderungen an die Cloudlösung sind so integriert, dass diese für jeden dargestellten Anwendungsfall der Industrie gewichtet werden können. Dabei wurden drei Kategorien ,weniger wichtig“, ,wichtig“ und ,sehr wichtig“ zur Bewertung herangezogen, die in der Abbildung durch Kreise in drei verschiedenen Größen dargestellt wurden.

Bei der Auswertung der Taxonomie zeigt sich, dass der Bedarf an einer Echtzeitverarbeitung die Anforderungen an die Cloudlösung erhöht. Zudem sind die Anforderungen an den Bereich Control höher als an den Bereich Monitoring, dies lässt sich durch den Eingriff ins System der durch den Steuerungsaspekt beschrieben ist erklären. So sind für die Auswertung von gerichtsverwertbaren oder auch historischen Daten Analysetools sehr wichtig. Des Weiteren ist in den beiden speziellen Anwendungen eine Integration von Big Data und Security wichtig. Gerichtsverwertbare Daten können z.B. im Smart City Bereich Umweltdaten sein. Für diese Art von Daten ist die Sicherheit, beispielsweise um eine Manipulation zu vermeiden, sogar sehr wichtig und könnte durch den Gebrauch von Blockchain unterstützt werden. Für Predictive Maintenance 
und Condition Monitoring sind die Anforderungen in Analyse, Big Data und IoT sehr hoch. In beiden Fällen sind die Kommunikation und Plug and Play wichtig, da Maschinen- und Produktionszustände von verschiedensten Anlagen und Sensoren übermittelt und zusammengefasst werden müssen. Damit so, in Verbindung mit den Analysetools eine Vorhersage über nötige Wartungen oder Produktveränderungen getroffen werden kann. Im Themenfeld Condition Monitoring kann eine Echtzeitüberwachung des Zustandes von Anlagen gefordert sein, dementsprechend sind die Anforderungen an den QoS und das SLA sehr hoch bzw. hoch. Immer wenn es darum geht Änderungen an Anlagen und/ oder Prozessen in Echtzeit umzusetzen, ist der Security Aspekt der Cloudlösung sehr wichtig, da z.B. bei einer zeitkritischen Steuerung die Prüfung auf Manipulation der Daten kaum umsetzbar ist. Im Rahmen einer Live-Überwachung, die ebenfalls einen Bedarf an Echtzeitverarbeitung hat, sind die Cloud-Komponenten Analyse, Big Data, IoT, QoS, Security, SLA und pay-as-you-go sehr wichtig und die Skalierbarkeit, Protokolle, Flexibilität und Blockchain wichtig. Dies spiegelt die Zunahme der Anforderungen an Cloudlösungen im Bereich der Echtzeitverarbeitung wieder. Die Daten müssen direkt, in Analysetools, die Big Data geeignet sind ausgewertet werden. Hierfür ist ein Anspruch an Latenzzeit, Bandweite und die allgemeine Performance gestellt und die Verfügbarkeit muss zu jedem Zeitpunkt über ein SLA gewährleistet sein. Ein ähnliches Anforderungsprofil lässt sich auf Seiten des Control Bereiches erkennen. Für den Basisanwendungsfall Monitoring ist für alle betrachteten spezifischen Anwendungen eine hohe Anforderung an Safety weniger wichtig. Für den Bereich Control werden dahingegen in den Anwendungen Motion Control, zeitkritische und sicherheitsrelevante Steuerung der Aspekt Safety als sehr wichtig betitelt. Es müssen Sicherheitsmechanismen einwandfrei funktionieren, sodass diese in automatisierten und flexibel gestalteten Prozessen, wenn notwendig, stets greifen. Die Anwendung Motion Control stellt zudem den Fokus in den Anforderungen an eine Cloud Lösung insbesondere auf QoS, Security, Verfügbarkeit und Skalierbarkeit. In der Bewegungssteuerung müssen exakte Positionierungen realisiert und Ansteuersignale gegeben werden, sodass eine Verfügbarkeit des Systems als auch die Anforderungen an den QoS zwingend notwendig ist. Diese Struktur bildet sich auch in der zeitkritischen Steuerung ab. Insgesamt werden im Anwendungsfall der zeitkritischen und sicherheitsrelevanten (Safety) Steuerung 58\% der Anforderungen als sehr wichtig, 33\% als wichtig deklariert. Damit sind die Anforderungen an diese Anwendungsfälle aus dem Themenkomplex Control vergleichbar mit denen der live Übertragung. 
Basisanwendungsfälle:
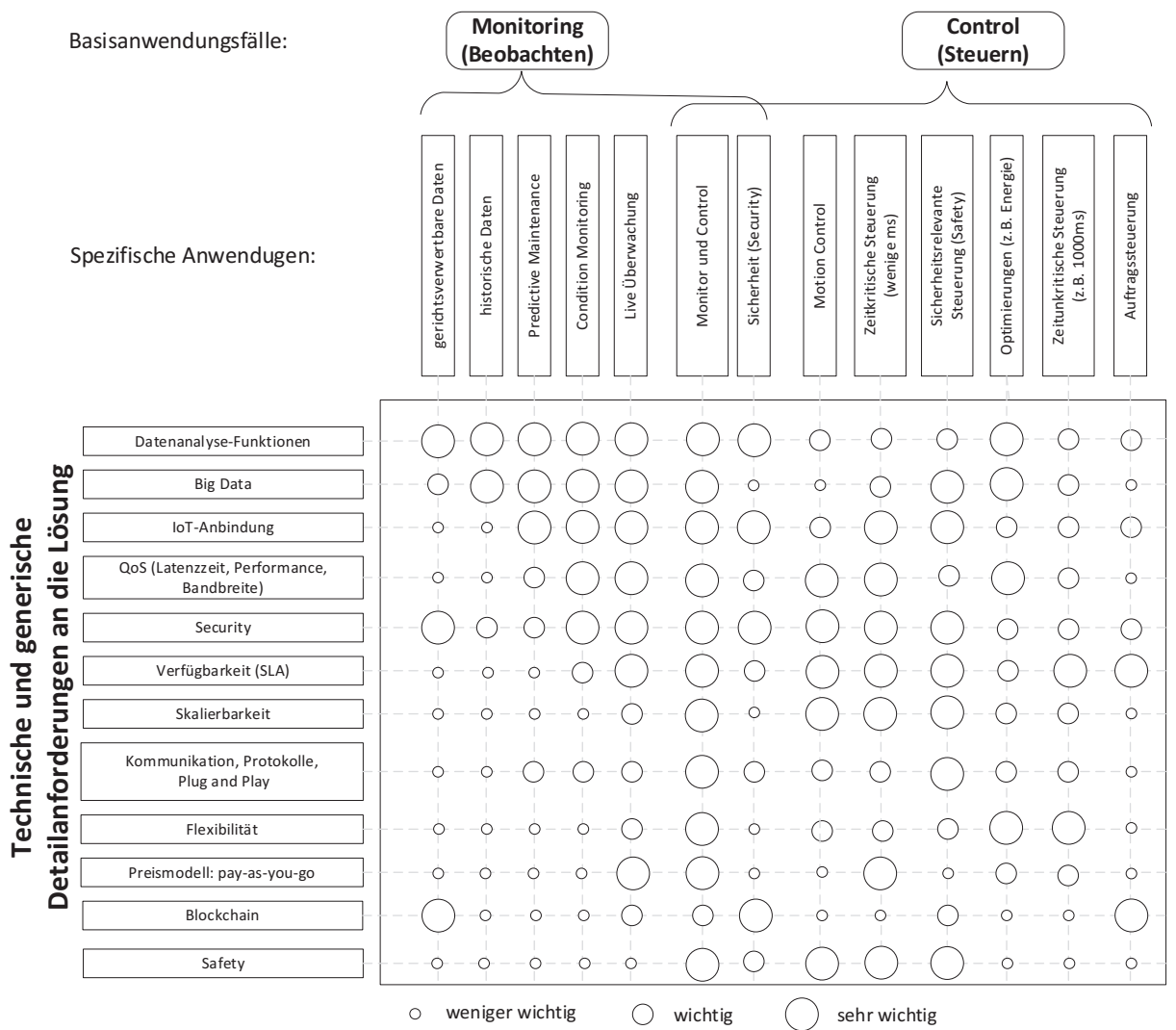

Abb. 2. Anforderungstaxonomie an industrielle Cloudinfrastrukturen: Sichtbar werden die Heterogenität und die Abhängigkeiten

In der flexiblen Produktion werden stetig Optimierungen und Anpassungen in Prozessen vorgenommen, diese stellen eine hohe Anforderung an die Analyse und Verarbeitung von Big Data, QoS und Flexibilität. Es müssen permanent zu optimierende Werte, wie die Energieeffizienz oder aber Wartezeiten analysiert werden und möglichst in Echtzeit auf die Ergebnisse dieser Analysen reagiert werden, sodass die Flexibilität der Produktion ausgeschöpft wird. Für die zeitunkritische Steuerung werden alle Anforderungen an die Cloud, außer Blockchain und Safety, als wichtig eingestuft. Trotz des unkritischen Zeitfensters und damit keinem Anspruch auf Echtzeit müssen die korrekten und relevanten Werte des Steuerungsprozesses bestimmt und übermittelt werden, denn Präzision und Sicherheit sind in dem Umfeld von äußerster Bedeutung. In den Basisanwendungsfällen ist auch der Sicherheitsaspekt in Bezug auf Security aufgeführt, da zur Gewährleistung der Security ebenfalls Anforderungen an Cloud Lösungen gestellt werden. So müssen durch Analysen sichergestellt werden, dass z.B. manipulierte Daten erkannt werden und im Bereich IoT z.B. eine sichere Kommunikation der Sensoren und Gateways mit den Maschinen umgesetzt wird. Weniger wichtig sind im Zusammenhang mit Security die Skalierbarkeit, die Flexibilität und das Preismodell. 
Die größte Anforderung an Cloud Lösungen stellt die Verbindung von Monitoring und Control. Es können die beiden Anwendungsfälle live Überwachung und zeitkritische Steuerung in Kombination auftreten. Das bedeutet, dass beispielsweise auf Grund einer live Überwachung in Echtzeit ein direkter Rückschluss auf Steuerungsmechanismen, die vorgenommen werden sollen, getroffen werden muss. Es sind alle Bereiche von der Analyse, über die garantierte Verfügbarkeit durch das SLA bis hin zur Kommunikation zwischen Sensorik, die die live Überwachung von Parametern umsetzen und Maschinen deren Steuerung auf Grund der Daten getriggert wird betroffen. Auf Grund der Control-Komponente in dieser spezifischen Anwendung wird die Anforderung an Safety in dem Zusammenhang als sehr wichtig eingestuft.

Zusammenfassend lässt sich in der Grafik in Abbildung 3 erkennen, dass die Anforderungen an Analyse, Big Data, IoT, SLA und Security in ihrer Wichtigkeit in Basisanwendungsfällen der Industrie dominieren. Es ist zu entnehmen, dass diese Anforderungskriterien in mindestens $50 \%$ der Fälle für die Anwendungsfälle mit sehr wichtig eingestuft wurden. Daran knüpfen die Anforderung an QoS, Skalierbarkeit, Flexibilität, Kommunikation, Protokolle und Plug and Play an. Diese Anforderungen werden jeweils in Summe mindestens 50\% der Fälle als sehr wichtig und wichtig eingestuft. Preismodelle, Blockchain-Technologien und Safety sind als Anforderungen an industrielle Cloudlösungen im Vergleich weniger wichtig, denn hier wurde in über $50 \%$ der Fälle die Anforderung als weniger wichtig evaluiert.

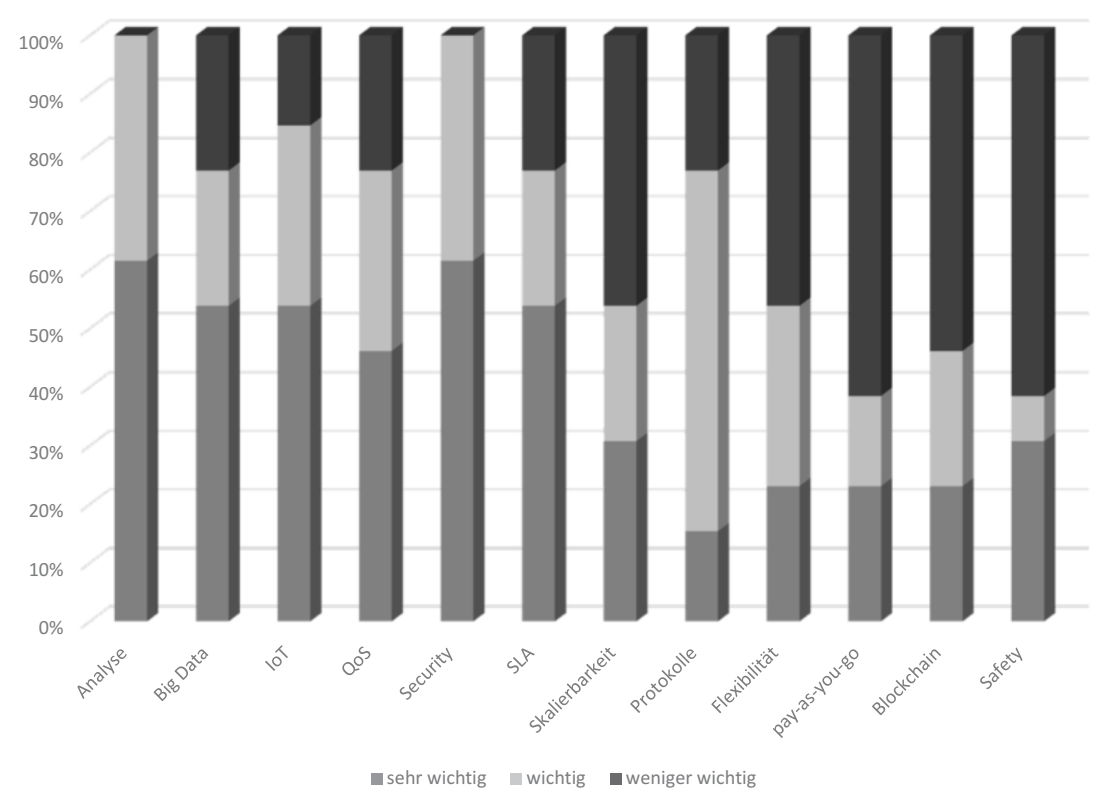

Abb. 3. Auswertung der Relevanz von Anforderungen an Cloud Lösungen 


\section{Cloud-Beispiele aus der industriellen Produktion und der Smart City und Einordnung in die Taxonomie}

Als Evaluation der Taxonomie werden zwei Beispiele aus industriellen Produktion und der Smart City beschrieben. Neben der Industrie ist Smart City ein Cloud- und IoTAnwendungsbereich, in dem die Anzahl an Sensoren und Aktoren, die vernetzt agieren, immer weiter steigt. In vielen Punkten stimmen die Anforderungen der beiden Bereiche überein. Zum Beispiel sind für eine durch Sensorik und Aktorik geschalteten Ampel die Echtzeitfähigkeit und Sicherheit unerlässliche Anforderungen an eine Cloud. Es dürfen keine falschen oder zu späten Ampelphasenschaltungen vorkommen, die die Verkehrssicherheit gefährden könnten. Dies spiegelt sich in der Produktion in zeitkritischen Steuerungen und sicherheitsrelevanten Steuerungen, die Menschen unter dem Safetyaspekt oder die Produktion unter dem Securityaspekt gefährden, wieder.

\subsection{Auftragssteuerung im IIC-Testbed Smart Factory Web}

Das IIC-Testbed Smart Factory Web wird vom Fraunhofer IOSB (Karlsruhe und Lemgo) gemeinsam mit dem koreanischen Partner KETI betrieben. Ziel ist es, basierend auf dem industriellen Internet der Dinge(IIoT) und Standards wie OPC UA und AutomationML Architekturen und Technologien für ein Netz verteilter Smart Factories zu realisieren und zu evaluieren. Das Smart Factory Web zielt darauf ab, ein Netzwerk intelligenter Fabriken mit flexibler Anpassung der Produktionskapazitäten und der gemeinsamen Nutzung von Ressourcen zu bilden, um die Auftragsabwicklung zu verbessern und auf Basis von Datenanalysen die Effizienz der Fabriken zu steigern. Die folgende Abbildung 4 zeigt die bisher vernetzten Smart Factories.

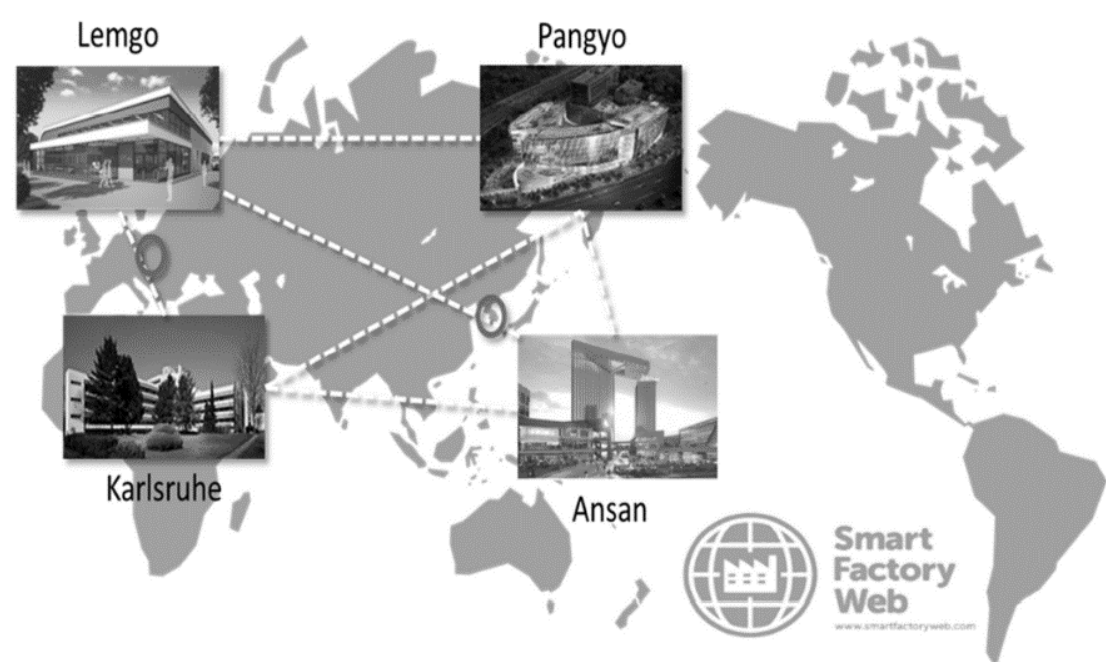

Abb. 4. Smart Factory Web: Vier vernetzte Smart Factories (Südkorea und Deutschland) 
Die einzelnen Maschinen und Anlagen der Smart Factories werden über einen Smart Factory Web Cloud Coupler mit integriertem OPC UA Aggregation Server mit einer Microsoft-Azure-Cloud-basierten Webportal verbunden [13]. Die folgende Abbildung 5 zeigt dies am Beispiel eines modularen Montagesystems der SmartFactoryOWL. Mittels AutomationML und OPC UA-Informationsmodell wurden die Fähigkeiten der Maschine Modelliert und die Produktionsressource in der Cloud (dem Smart Factory Web) zu Verfügung gestellt. Im Smart Factory Web kann nun für spezifische Produktproduktionsbedarfe weltweit nach einer passenden Produktionsstätte gesucht werden und diese dann auch gebucht werden. Diese Buchung soll in Zukunft vertragssicher (Blockchain) erfolgen.

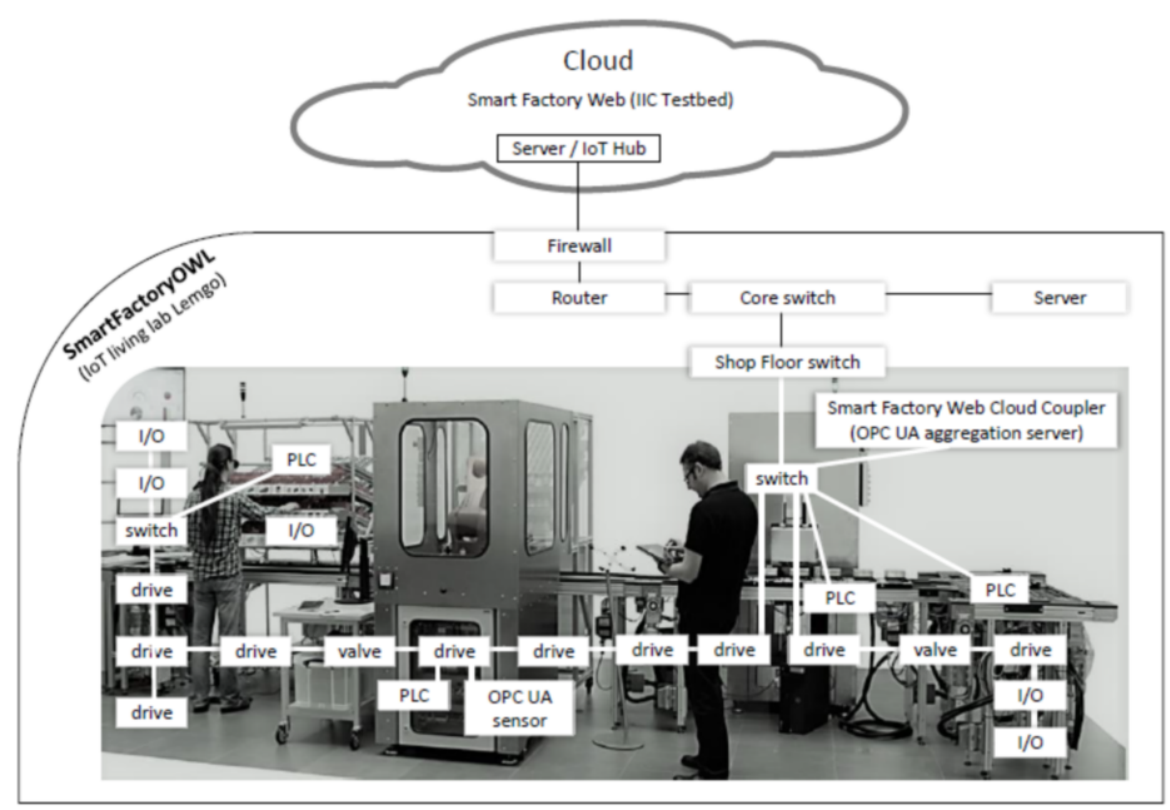

Abb. 5. Koppelung einer Maschine mit der SmartFactoryOWL mit der Cloud 


\subsection{Beispiel eines Use Case der Smart City im Cloud und IoT- Anwendungsbereich}

In Smart City-Projekten wird häufig Echtzeit-Geotracking des öffentlichen Personennahverkehrs umgesetzt, um den Bürgern und Fahrgästen einen Mehrwert zu bieten und eine Grundlage für weitere Anknüpfungspunkte zu schaffen. Die Echtzeit-Information der Busposition gibt Fahrgästen einen Überblick über Verspätungen und Abfahrtssituationen an Haltestellen. In der Zukunft werden Daten und Mehrwerte, die im Bereich des Echtzeit-Geotracking des öffentlichen Personennahverkehrs aufgezeichnet und erzeugt werden, ausgewertet. So können mit Analysetools die Bewegungsdaten von Bussen ausgewertet und ein Indiz für die Verkehrslage in der Stadt geben. Wenn z.B. Busse verspätet sind oder sich nur mit einer geringen Geschwindigkeit fortbewegen, ließe sich daraus mit einer gewissen Wahrscheinlichkeit prognostizieren, dass die Verkehrsauslastung der Straße hoch sein muss. Anhand dieses Beispiels wird die Anforderung an die Cloud Lösung im Bereich der Analyse deutlich. Es können auch weitere Informationsknoten, wie z. B. der Zustand von Bahnschranken, welche auf der Buslinie liegen integriert werden und so bei längeren Schließzeiten der Schranken dem Bus eine alternative Routenführung vorschlagen um die Verspätung zu minimieren. Somit würde eine Vernetzung der Busposition mit weiteren Sensorknoten in der Stadt zusätzliche Usecase und Mehrwerte bieten. Besonders bezogen auf Smart City, sind Kommunen darauf bedacht, dass keine personenbezogenen und sensiblen Daten nach außen gelangen und entsprechende Security Vorkehrungen getroffen werden. Insbesondere dürfen Daten, die in der Öffentlichkeit gezeigt werden, nicht manipuliert werden und entsprechend gegen Cyber Angriffe geschützt werden. Dies kann sogar so weit gehen, dass Safetyrelvante Situationen z.B. Ampelkreuzungen, die intelligent den Verkehr regeln, abgebildet werden und natürlich einen sehr hohen Anspruch an die Security der Cloud stellen. Dieses Beispiel der Ampelschaltung verdeutlicht auch den Anspruch an ein SLA; denn Ausfälle der Systeme sollten möglichst vermieden werden und über eine Verfügbarkeitsgarantie abgedeckt werden. Das Gleiche gilt für die Anforderung an QoS, da Echtzeitfähigkeit für Geotracking von bewegten Objekten entscheidend ist. Bei einer Geschwindigkeit von $50 \mathrm{~km} / \mathrm{h}$ werden bereits $500 \mathrm{~m}$ in $38 \mathrm{~s}$ zurückgelegt. Das heißt ein Geotracking von bewegten Objekten mit einem Intervall von z. B. $30 \mathrm{~s}$ liefert nicht die benötigte Auflösung. Dieses Beispiel zeigt die Vielseitigkeit eines Smart City Use Case und den Gedanken der globalen Vernetzung der verschiedensten Objekte, wie z. B. Bus, Ampel und Bahnschranke in den Städten und die damit einhergehenden Anforderungen an Cloud Lösungen.

\section{$5 \quad$ Zusammenfassung und Ausblick}

In diesem Paper wurde eine Anforderungstaxonomie mit Bezug auf Basisanwendungsfälle für industrielle Cloud Lösungen erstellt. In diesem Zusammenhang wurden technische und generische Detailanforderungen, die an Cloud Lösungen gestellt, herausgearbeitet und hinsichtlich spezifischer Anwendungsfälle evaluiert. In dem dreistufigem Bewertungsverfahren zeigte sich, dass Anwendungen, die eine Echtzeitverarbeitung oder eine nahezu Echtzeitverarbeitung voraussetzten, die höchsten Anforderungen 
an die Cloud stellen. Zudem zeigt die Taxonomie, dass sich die Bereiche Datenanalyse und Security hervorheben, da diese Anforderung stets als wichtig bzw. sehr wichtig eingestuft wurde. Schließlich sind es aber auch Big Data und Verfügbarkeitsgarantie (SLA), die zusammen mit dem QoS sich zusätzlich zu Analyse und Security als die entscheidenden Anforderungen evaluieren ließen. Diese Aspekte wurden in zwei Beispielen aus Industrie und Smart City wiedergespiegelt und beschrieben. In einem nächsten Schritt müssten Anbieter von Cloud Plattformen zu der Taxonomie verknüpft werden, sodass der spezifischen Anwendung folgend ein Portfolio an geeigneten Plattformen vorgestellt wird. Hierzu muss eine Marktübersicht an Cloud Plattformen erstellt und entsprechend der Anforderungen bewertet werden.

\section{Literatur}

[1] Wollschläger, M.; Sauter, T.; Jasperneite, J.: The future of industrial communication. IEEE Industrial Electronics magazine. IEEE, März 2017.

[2] Schriegel, S.; Kobzan, T.; Jasperneite, J.: Investigation on a Distributed SDN Control Plane Architecture for Heteregenous Time Sensitve Networks. 14th IEEE International Workshop on Factory Communication Systems (WFCS) Imperia (Italy), Juni 2018.

[3] Schriegel, S.; Pethig, F.; Windmann, S.; Jasperneite, J.: PROFIanalytics - die Brücke zwischen PROFINET und Cloud-basierter Prozessdatenanalyse. Automation 2017 , Baden-Baden, Oktober 2017.

[4] ISO/IEC: Information technology - Internet of Things Reference Architecture (IoT RA). ISO/IEC CD 30141, Schweiz, 2016.

[5] Lassnig, M.; Schön S.; Stabauer, P.; Selhofer, H.: Transformation verschiedener Wirtschaftssektoren durch Industrie 4.0 - Wie sich ausgewählte Branchenprofile im Industrial Internet verändern. InnovationLab Arbeitsberichte, Salzburg Research Forschungsgesellschaft, 2017.

[7] Botta, A.; de Donato, W.; Persico, V.; Pescapé, A.: On the Integration of Cloud Computing and Internet of Things. International Conference on Future Internet of Things and Cloud (FiCloud), Spanien, 2014.

[6] World Economic Forum: Industrial Internet of Things: unleashing the potential of connected products and services. 2015.

[8] NIST Big Data Public Working Group, Definitions and Taxonomies Subgroup: NIST Big Data Interoperability Framework: Volume1: Definitions. National Institute of Standards and Technology Special Publication 1500-1, 2015.

[9] ISO/IEC JTC 1, Information Technology: Big data Preliminary Report 2014. ISO/IEC, Schweiz, 2015.

[10] Mell, P.; Grance, T.: The NIST Definition of Cloud Computing. National Institute of Standards and Technology Special Publication 800-145, 2011.

[11] Müller, A.: Radio communication for Industrie 4.0. ITG-expert comittee 7.2 radio systems, Robert Bosch GmbH 2015. 
[12] Fysarakis, K.; Askoxylakis, I.; Soultatos, O.; Papaefstathiou, I.; Manifavas, C.; Katos, V.: Which IoT Protocol? Comparing standardized approaches over a common M2M application. IEEE Global Communications Conference (GLOBECOM), Washington DC, USA, Februar 2016.

[13] Heymann, S.; Stojanovic, L.; Watson, K.; Seungwook, N.; Song, B.; Gschossmann, H.; Schriegel, S.; Jasperneite, J.: Cloud-based Plug and Work architecture of IIC Testbed Smart Factory Web. In: IEEE 23rd International Conference on Emerging Technologies and Factory Automation (ETFA). Torino, Italy, September 2018.

Open Access Dieses Kapitel wird unter der Creative Commons Namensnennung 4.0 International Lizenz (http://creativecommons.org/licenses/by/4.0/deed.de) veröffentlicht, welche die Nutzung, Vervielfältigung, Bearbeitung, Verbreitung und Wiedergabe in jeglichem Medium und Format erlaubt, sofern Sie den/die ursprünglichen Autor(en) und die Quelle ordnungsgemäß nennen, einen Link zur Creative Commons Lizenz beifügen und angeben, ob Änderungen vorgenommen wurden.

Die in diesem Kapitel enthaltenen Bilder und sonstiges Drittmaterial unterliegen ebenfalls der genannten Creative Commons Lizenz, sofern sich aus der Abbildungslegende nichts anderes ergibt. Sofern das betreffende Material nicht unter der genannten Creative Commons Lizenz steht und die betreffende Handlung nicht nach gesetzlichen Vorschriften erlaubt ist, ist für die oben aufgeführten Weiterverwendungen des Materials die Einwilligung des jeweiligen Rechteinhabers einzuholen. 